\title{
Gardening May Repeatedly Activate the Frontal Pole
}

\author{
Masahiro Toyoda1 ${ }^{*}$, Yuko Yokota1, Susan Rodiek ${ }^{2}$ \\ ${ }^{1}$ Graduate School of Landscape Design and Management, University of Hyogo, Awaji, Japan \\ ${ }^{2}$ Center for Health Systems \& Design, Texas A \& M University, College Station, TX, USA \\ Email: *masahiro_toyoda@awaji.ac.jp
}

How to cite this paper: Toyoda, M., Yokota, Y. and Rodiek, S. (2017) Gardening May Repeatedly Activate the Frontal Pole. Journal of Behavioral and Brain Science, 7, 464-483.

https://doi.org/10.4236/jbbs.2017.710033

Received: August 31, 2017

Accepted: October 8, 2017

Published: October 12, 2017

Copyright ( $) 2017$ by authors and Scientific Research Publishing Inc. This work is licensed under the Creative Commons Attribution International License (CC BY 4.0).

http://creativecommons.org/licenses/by/4.0/

(c) (i) Open Access

\begin{abstract}
Previous epidemiological studies have indicated the effectiveness of gardening and other activities for dementia prevention. Our previous study using near-infrared spectroscopy (NIRS) suggested seeding and watering tasks could induce greater activation than other gardening tasks in the prefrontal area of older adults, but repetitive effects were not fully addressed. The objective of this NIRS study was to investigate the effects of repeated gardening tasks on activation of the frontal pole (FP). We measured oxygenated hemoglobin $(\mathrm{Oxy}-\mathrm{Hb})$ values in the FP while 24 healthy right-handed older adults (60 - 73 years) performed a seeding task, a watering task, and a motor programming task (FAB 3) of the Frontal Assessment Battery (FAB). Each task was repeated five times. After the fourth trial, comparison of maximum Oxy-Hb values among the three tasks demonstrated that, in the medial FP, activation during the two gardening tasks was significantly or marginally significantly greater than activation during the FAB 3 task. The results may be attributable to differences in the number of sensory information sources and the number of stimulus-oriented (SO) attention switches and SO thoughts. No significant differences were observed in activation in the lateral FP. Comparison of maximum $\mathrm{Oxy}-\mathrm{Hb}$ values among five trials within each task showed sustained medial FP activation in all tasks, while the watering task and FAB 3 task showed significantly decreased activation in a portion of the lateral FP. This was possibly due to differences in the number of times switching between SO attention and stimulus-independent (SI) attention, and differences in frequency of changes of visual dimension weighting. Also, in the gardening tasks, the participants needed to respond to changes in the external environment; it can be speculated that the need for collating external information corresponding to changes in the external environment induced repeated FP activation during the gardening tasks.
\end{abstract}




\section{Keywords}

Near-Infrared Spectroscopy (NIRS), Stimulus-Oriented Attention, Visual Dimension Weighting, Eye-Hand Coordination, Dementia Prevention

\section{Introduction}

Although cognitive decline in people with mild cognitive impairment (MCI) and Alzheimer's disease (AD) is well-known, age-related decline of cognitive and executive functions may arise even in healthy older adults. Decline of the working memory (WM) function is considered to be one of the causes [1]. It is also reported that blood flow in the dorsolateral prefrontal cortex (DLPFC), which is closely related to WM, generally decreases in healthy older adults over time [2]. On the other hand, Zinke, Zeintl, Rose, Putzmann, Pydde, and Kliegel reported the effects of WM training in healthy older adults and suggested preserved cognitive plasticity over a large range of old age [3]. Benefits of training prefrontal lobe functions for suppressing cognitive decline and/or improving cognitive functions is also reported even in older adults with MCI [4]. These studies suggest that activities stimulating frontal lobe functions and appropriate training can be effective and have the potential to contribute to maintenance and/or improvement of cognitive functions in a wide range of the population.

In addition, some epidemiological studies have indicated the effectiveness of physical, social and leisure activities for dementia prevention, some of which include gardening activities [5] [6] [7]: Simons, Simons, McCallum, and Friedlander recommended the continuation of daily gardening in the hope of reducing the incidence of dementia in future years. They indicated the strong protective effect of daily gardening against the onset of dementia as a physical activity, and appropriateness as a leisure or mental activity in senior citizens as well [6]. Jedrziewski, Ewbank, Wang, and Trojanowski reported a significant protective effect of gardening/yard work with sessions lasting at least 20 minutes, and indicated the potential to lower the risk of dementia. Furthermore, they suggested the strong association between decreased risk of dementia and the number and diversity of physical activities pursued; they also mentioned additional benefits from socialization and cognitive stimulation attributed to gardening/yard work [7].

There is a huge variety of possible gardening activities. However, most epidemiological studies indicating the effectiveness of gardening activities did not specify what kinds of gardening activities the study participants actually engaged in. If gardening activities are effective for dementia prevention, many of them might have some common features leading to cognitive stimulation. Seeding, thinning, watering, weeding, planting seedlings, and harvesting are typical gardening activities. We speculated that such gardening activities would have "motor programming" and "repeated motions" in common. 
In a previous relevant study using near-infrared spectroscopy (NIRS), we investigated the effects of a motor programming task itself on the activation of the prefrontal cortex (PFC) in healthy adults. In the study [8], we compared PFC activation among three age groups (the younger group: 20 - 39 years, the middle-aged group: 40 - 59 years, and the older group: 60 - 81 years) during three tasks selected out of the Frontal Assessment Battery (FAB): a motor programming task (FAB 3), a sensitivity-to-interference task (FAB 4), and an inhibitory control task (FAB 5). The FAB is a standardized measure battery of frontal lobe functions consisting of six subtests examining conceptualization, mental flexibility, motor programming, sensitivity to interference, inhibitory control, and environmental autonomy [9]. In performing FAB 3, a participant imitates, memorizes, and conducts a series of three hand motions (fist-edge-palm) with his/her right hand on his/her left palm. In FAB 4 and FAB 5, a participant responds to the examiner's instructions by the action of tapping his/her finger on the desk. All these three tasks are accompanied by hand motions. In our study, the activation in the frontal pole (FP) and the DLPFC during the motor programming task (FAB 3) was found to be greater than or comparable with that found during the other two tasks (FAB 4 and FAB 5) in the group of older adults over 60. Repetition of a series of hand motions and attention to action were speculated to have caused such activation [8].

In our subsequent NIRS study [10], we investigated the effects of gardening activities on PFC activation. We selected five gardening tasks (seeding, thinning, weeding, planting seedlings, and watering), as typical gardening tasks. PFC activation in the FP and the DLPFC during all these gardening tasks was found to be greater than or similar to that during a control task (a motor programming task [FAB 3]) in younger adults (20 - 59 years). However, in older adults (60 - 88 years), greater activation was observed in the DLPFC during a seeding task, and in the FP during a watering task, compared to the control task. In summary, the seeding task and the watering task activated the FP and the DLPFC in both age groups. Although these two gardening tasks and the control task had common elements of "motor programming" and "repetition of motions", the two gardening tasks seemed to have additional elements related to PFC activation.

In the study, participants repeated each task three times. However, in actual gardening settings, it is assumed that there will be a greater number of task repetitions, and the working hours will be longer than in experimental settings. As Strange, Henson, Friston, and Dolan noted, the FPPC [fronto-polar prefrontal cortex] is engaged during intentional or explicit rule induction, but once a rule is learned, more posterior areas mediate rule application [11]. According to their findings, repetition of the same tasks might not lead to continuing activation in the same brain area. In other words, although three trials of the gardening task caused activation in the FP and DLPFC, the question still remained: Would the same gardening task repeated several times show continuing PFC activation?

The FP is known to be involved in human cognitive processing such as recollection of source information [12], episodic memory retrieval [13], prospective 
memory [14], metacognition (evaluation, monitoring, or manipulation of internally generated information) [15], cognitive branching [16], a default state of brain activity [17], and coordination of internally- and externally-generated information [18] [19]. In addition, in their retrieval model, Fletcher and Henson attributed higher-level control processes (i.e. the selection of processes/subgoals) to the anterior frontal cortex (AFC) [20].

The gardening tasks in the study, which activated the FP and the DLPFC, can also be thought of as the tasks requiring higher-level control processes. From the perspectives of the effectiveness of gardening activities for dementia prevention, as shown in epidemiological studies and gardening-related cognitive stimulation, we hypothesized that the activation in the FP involved in cognitive processing would be maintained even when gardening tasks such as seeding and watering were repeated several times. In the current NIRS study presented in this paper, we investigated activation in the FP in healthy older adults without dementia, while they were repeating a seeding task and a watering task five times respectively.

\section{Materials and Methods}

\subsection{Participants}

Through the University of Hyogo website, we recruited right-handed healthy older adults with ages ranging between 60 and 74, and having a background of gardening experience of more than one year. The reason we exclusively recruited people with some gardening experience was that FP activation during gardening tasks might be affected by the presence or absence of gardening experience in the participants. Specifically, the participants without experience would perform the gardening tasks as first-time tasks to be learned, while the participants with experience would perform the tasks based on their past experience. Concerning the age range, the lower age limit of 60 was based on a report of rapid decline of executive function after age 60 [21]; the upper age limit of 74 was set according to the age boundary between "young-old (65 - 74 years)" and "old-old (75 years or over)" in the medical care system for the elderly in Japan. The number of applicants was twenty-four, all of whom were accepted for the study; they included 9 men $(\mathrm{M}=67.7$ years, $\mathrm{SD}=1.7$, range: $65-70$ years $)$ and 15 women $(\mathrm{M}=65.5$ years, $S D=4.0$, range: $60-73$ years) (Table 1 ). Their right-handedness was orally confirmed during the recruiting process.

All participants received a detailed explanation of the study such as objectives, methods, prospective risk, and privacy policy in advance; all provided written

Table 1. Demographics of the participants.

\begin{tabular}{ccccc}
\hline & $n$ & mean age (years) & SD & range (years) \\
\hline Male & 9 & 67.7 & 1.7 & $65-70$ \\
Female & 15 & 65.5 & 4.0 & $60-73$ \\
Total & 24 & 66.0 & 3.4 & $60-73$ \\
\hline
\end{tabular}


informed consent. The research protocol was approved by the Ethics Committee in the Graduate School of Landscape Design and Management, the University of Hyogo.

\subsection{Tasks}

This study was conducted in a quiet classroom (room size: approximately 60 square meters) at the University of Hyogo for five days between late February and March 2016 (average temperature: $20.0^{\circ} \mathrm{C}$, average humidity: $37.5 \%$ ).

The participants entered the room individually, and performed three tasks in a sitting posture with NIRS optodes positioned on the head. A NIRS optode is an optical sensor device to measure the local changes in oxygenated hemoglobin $(\mathrm{Oxy}-\mathrm{Hb})$, deoxygenated hemoglobin (Deoxy-Hb) and total hemoglobin (Total-Hb) in the PFC.

The research tasks were a seeding task and a watering task, as well as the FAB 3 , a control task. In the seeding task, the participant was required to sow common beans (Phaseolus vulgaris, approximately $13 \mathrm{~mm} \times 7 \mathrm{~mm}$ ) into a square tray that had 25 cells, each measuring $55 \mathrm{~mm} \times 50 \mathrm{~mm} \times 50 \mathrm{~mm}$. The process was to make a hole in the center of each cell with a forefinger, pick up a seed with a forefinger and a thumb, put the seed into the hole, gather the soil around the hole, and press the surface of the soil with a forefinger. The watering task was to water the soil in cells of the same type of a square tray where common beans had already been sown. Watering was conducted by using a plastic bottle of $500 \mathrm{ml}$ in capacity $(65 \mathrm{~mm}$ across $\times 220 \mathrm{~mm}$ high) with a sprinkling nozzle. The participant did not need to squeeze the plastic bottle because water ran out through the sprinkling nozzle just by tilting the bottle. The cells for the watering task had been filled with soil in advance; the level of soil in each cell was $10 \mathrm{~mm}$ lower than the brim, to provide enough watering space. The direction of work was from the top left cell to the bottom right cell; that is, the participant was required to start the task at the top left cell, move rightward to the end of the row and move to the left-most cell of the next lower row. The control task was FAB 3 , a motor programming task repeating a series of three hand motions (fistedge-palm) on the palm (Figure 1).

Before starting the seeding task and the watering task, the participant was given an explanation of the tasks with a demonstration by the examiner; each person had one chance to try out each task. In demonstrating the FAB 3 task, the examiner first showed three series of hand motions (fist-edge-palm), according to Dubois' procedure [9], after which the participant repeated the series of motions three times. The examiner asked participants not to close their eyes in conducting the FAB 3 task.

\subsection{Procedure}

Five trials were run for each task. The order of the three tasks was the control task (FAB 3), the seeding task, and the watering task. The protocol was as 


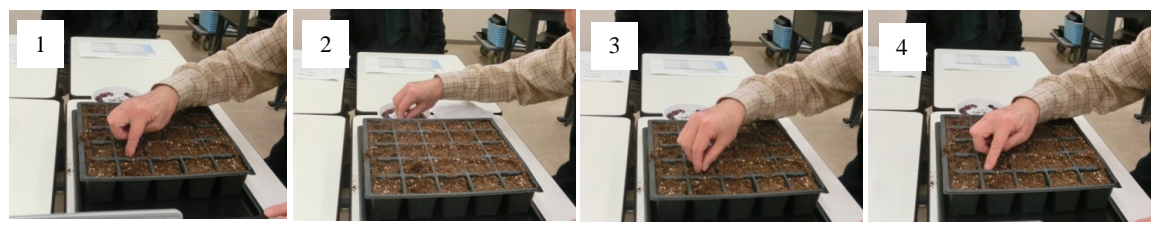

(a)

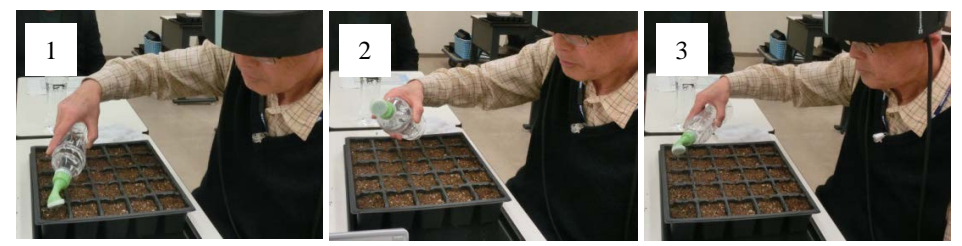

(b)
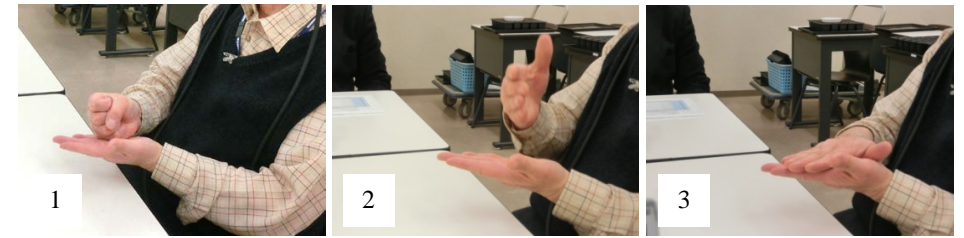

(c)

Figure 1. The process of two research tasks and a control task. The upper two rows of pictures show the process of two gardening tasks and the bottom row shows the process of the control task (FAB 3). (a) Seeding task: 1 . Make a hole $\rightarrow 2$. Pick up a seed $\rightarrow 3$. Put the seed into the hole $\rightarrow 4$. Gather the soil around the hole; (b) watering task: 1 . Water the soil in a cell $\rightarrow 2$. Raise the plastic bottle $\rightarrow 3$. Water the soil in the next cell; (c) control task (FAB 3): 1. Fist $\rightarrow$ 2. Edge $\rightarrow$ 3. Palm.

follows: positioning the head unit of NIRS, instructions and practice for FAB 3 (60 seconds), the baseline period (15 seconds), (FAB 3 [15 seconds] and rest [15 seconds] $) \times 5$ sets, instructions and demonstration for the seeding task (60 seconds), the baseline period (15 seconds), (the seeding task [15 seconds] and rest $[15$ seconds] $) \times 5$ sets, instructions and demonstration for the watering task(60 seconds), the baseline period (15 seconds) and (the watering task [15 seconds] and rest [15 seconds]) $\times 5$ sets (Figure 2). Each instruction included demonstration by the examiner and practice by the participant. The number of practice sessions was three times for the FAB 3, and one time each for the seeding task and the watering task. During task performance, participants were instructed not to move their heads widely. During the baseline period and the rest period, participants were required to gaze blankly at a double circle on a piece of white paper in front of them at a distance of 1 meter, so they could recover enough to be stable. During task performance, we observed whether participants were watching their hand motions and materials such as cells, soil, seed, and water, or not.

\subsection{NIRS Measurements}

NIRS is a noninvasive tool to examine brain activation while participants are performing activities in a sitting posture. We measured relative changes in the 


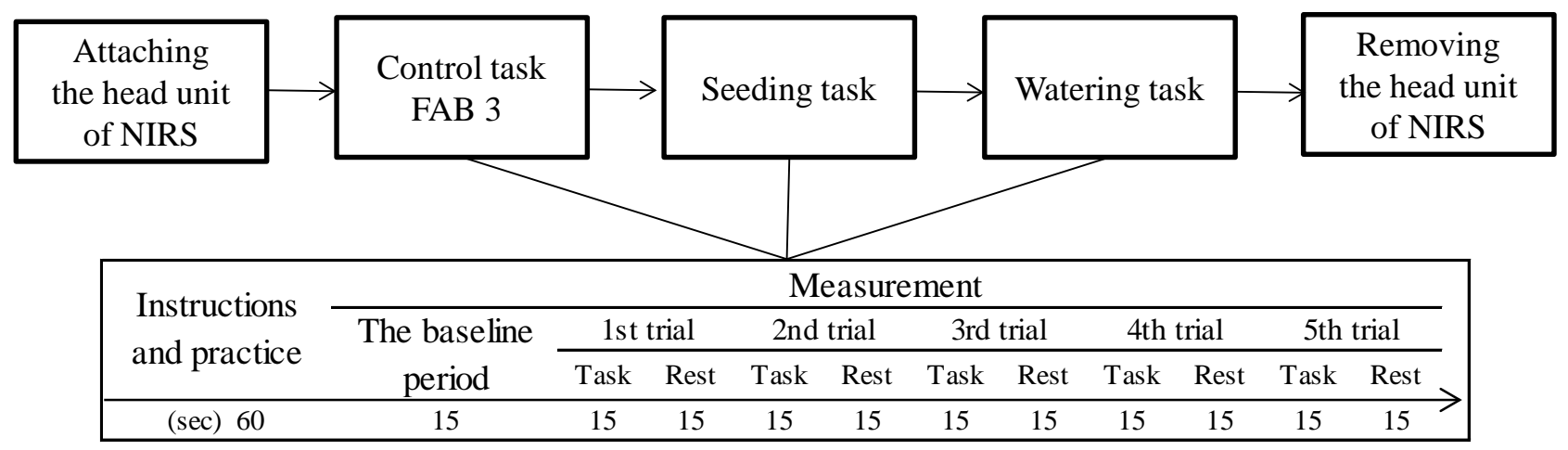

Figure 2. The protocol. Each task was conducted according to the same flow. NIRS = Near Infrared Spectroscopy; FAB $=$ the Frontal Assessment Battery.

concentration of Oxy-Hb, Deoxy-Hb, and Total-Hb during the tasks by using a 16-channel (CH) functional NIRS equipment, Spectratech OEG-16, (Spectratech Inc., Tokyo). The equipment has 16 simultaneous measurement channels and uses two wave-lengths of near-infrared light $(770 \mathrm{~nm}$ and $840 \mathrm{~nm})$. The constituents of an OEG-16 sensor band are light-emitting parts of 6 built-in type LEDs with two waves and light-receiving parts of 6 photo diodes. Each channel (i.e. measuring point) consists of a pair of optodes -1 emitter and 1 detector-with a distance between them of $30 \mathrm{~mm}$ (Figure 3). Absorption of near-infrared light was measured with a sampling interval of 0.65 seconds. In attaching the head unit onto a participant's forehead, the center of the sensor band was placed on the Fpz according to the International 10 - 20 system, a standard for electroencephalography (EEG) electrode positioning [22]. The sensor band was covered with a headband in order to make sure that all optodes contacted the skin of the forehead of the participant. We used the variations in the Oxy-Hb concentration as indicators of changes in the regional cerebral blood flow ( $\mathrm{rCBF}$ ), because $\mathrm{Oxy}-\mathrm{Hb}$ was considered to be more sensitive than Deoxy- $\mathrm{Hb}$ as a parameter for measuring the blood flow relevant to PFC activation, as Hoshi, Kobayashi, and Tamura noted [23].

The regions measured by fNIRS in our study included the FP, the DLPFC, the inferior frontal gyrus (including the orbitofrontal cortex), the pars triangularis Broca's area, and the frontal eye field. We focused on the FP and used the data of $\mathrm{CH} 4-6$ as the right lateral FP, $\mathrm{CH} 7$ as the right medial FP, $\mathrm{CH} 10$ as the left medial FP, and CH $11-13$ as the left lateral FP.

\subsection{Data Analysis}

We compared task performance and Oxy-Hb values among three tasks by trial, and Oxy-Hb values among five trials by task. Task performance of the seeding task and the watering task was indicated by the number of completed cells at each trial. Unfinished cells were not counted. Task performance of the control task was indicated by the number of series of motions completed.

For data analysis, we used the maximum $\mathrm{Oxy}-\mathrm{Hb}$ value (from the baseline level during each period of task performance rather than the average value, 


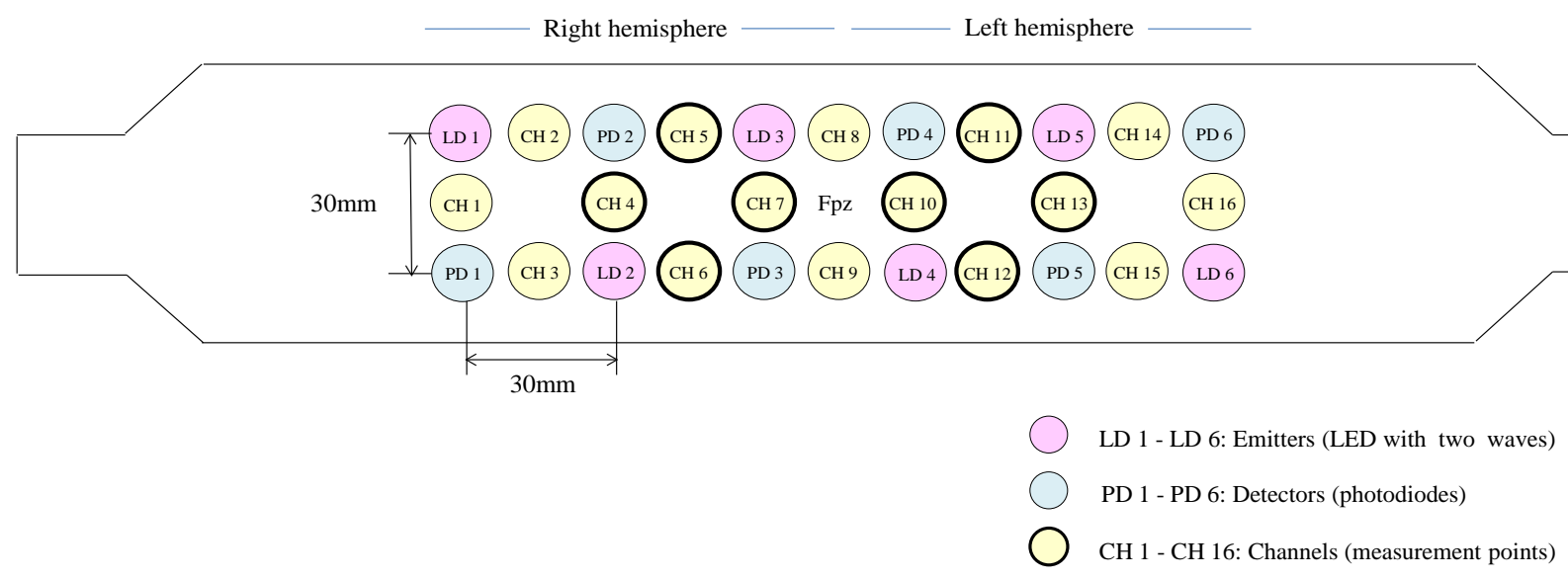

Figure 3. Location of 12 optodes (6 emitters and 6 detectors) and 16 measurement points (channels) in theOEG-16 head module. One channel consists of 1 emitter and 1 detector at a distance of $30 \mathrm{~mm}$ from each other. We focused on the channels encircled by heavy lines.

based on the findings of previous studies). Sakatani, Xie, Lichty, Li, and Zuo had reported that the NIRS parameters tended to return toward the preactivation baseline after reaching the maximum value, or to fluctuate during the task, possibly because of attention changes [24]. Gilbert, Frith, and Burgess mentioned transient activity of right rostrolateral PFC in switching between stimulus-oriented (SO) and stimulus-independent (SI) thought [25]; Burgess reported transient activity exhibited in the rostral PFC in switching between two phases [19]. In analyzing our findings, statistical analyses were performed using Excel 2010 (Microsoft, USA) with the add-in software SSRI, Version 1.02, 2012, (Social Survey Research Information Co., Ltd.), with $p<0.05$ considered statistically significant.

\section{Results}

\subsection{Direction of Participants' Eyes during Task Execution}

From the results of observation, nineteen of twenty-four participants (79.2\%) executed the FAB 3 task without looking at their hands during any of five trials. In contrast to this, all the participants executed the seeding task and the watering task while looking at their hand motions and at the elements necessary to accomplish the tasks, such as cells, soil, seeds, and running water.

\subsection{Task Performance}

Table 2 shows changes in task performance of three tasks with the increased number of trials. For all three tasks, median values in each trial were similar, or larger, compared with the values in the trial that had just been completed. In FAB 3, the Steel-Dwass test for multiple comparison within the task indicated significant differences $(p<0.05)$ between the first trial and the third trial $(t=$ 2.779, $p=0.043)$, between the first and the fourth trials $(t=3.814, p=0.001)$, between the first and the fifth trials $(t=4.444, p<0.001)$, and between the 
Table 2. Task performance in all trials, for the three tasks. Task performance of FAB 3 is rated by the number of completely finished series of motions, and that of two gardening tasks is rated by the number of finished cells. Unfinished cells were not counted. Mdn = median; Q1 = the first quartile; Q3 = the third quartile. The values with ${ }^{\star}$ showed significant differences as compared with the 1 st trial.

\begin{tabular}{|c|c|c|c|c|c|c|}
\hline Tasks & & 1st trial & 2nd trial & 3rd trial & 4th trial & 5th trial \\
\hline \multirow[t]{6}{*}{ FAB 3} & $\mathrm{n}$ & 24 & 24 & 24 & 24 & 24 \\
\hline & Min & 4.00 & 4.00 & 5.00 & 5.00 & 5.00 \\
\hline & Q1 & 5.00 & 5.75 & 5.75 & 6.00 & 6.00 \\
\hline & Mdn & 5.00 & 6.00 & $6.50^{*}$ & $7.00^{*}$ & $7.00^{*}$ \\
\hline & Q3 & 6.00 & 7.00 & 7.00 & 8.00 & 8.25 \\
\hline & $\operatorname{Max}$ & 8.00 & 11.00 & 12.00 & 13.00 & 13.00 \\
\hline \multirow[t]{6}{*}{ Seeding } & $\mathrm{n}$ & 24 & 24 & 24 & 24 & 24 \\
\hline & Min & 1.00 & 2.00 & 2.00 & 2.00 & 2.00 \\
\hline & Q1 & 2.00 & 2.00 & 2.00 & 2.00 & 2.00 \\
\hline & Mdn & 2.00 & 2.00 & $2.50^{*}$ & $3.00^{*}$ & $3.00^{*}$ \\
\hline & Q3 & 2.00 & 3.00 & 3.00 & 3.00 & 3.00 \\
\hline & Max & 3.00 & 4.00 & 4.00 & 4.00 & 4.00 \\
\hline \multirow[t]{6}{*}{ Watering } & $\mathrm{n}$ & 24 & 24 & 24 & 24 & 24 \\
\hline & Min & 2.00 & 3.00 & 3.00 & 3.00 & 3.00 \\
\hline & Q1 & 3.00 & 4.00 & 4.00 & 5.00 & 5.00 \\
\hline & Mdn & 4.00 & 5.00 & $5.00^{*}$ & $5.00^{*}$ & $5.00^{*}$ \\
\hline & Q3 & 5.00 & 7.00 & 7.00 & 7.00 & 7.25 \\
\hline & Max & 8.00 & 12.00 & 10.00 & 12.00 & 10.00 \\
\hline
\end{tabular}

second and the fifth trials $(t=2.928, p=0.028)$. In the seeding task, there were significant differences between the first and the third trials $(t=3.075, p=0.018)$, between the first and the fourth trials $(t=3.735, p=0.002)$, between the first and the fifth trials $(t=4.182, p<0.001)$, and between the second and the fifth trials $(t$ $=2.808, p=0.040)$. In the watering task, there were significant differences between the first and the third trials $(t=2.827, p=0.038)$, between the first and the fourth trials $(t=3.371, p=0.007)$, and between the first and the fifth trials $(t=$ $3.529, p=0.004)$.

The time required to complete a single series of motions in each trial was calculated by using a median of task performance (i.e. 15 [seconds] divided by a median). The first trial of the FAB 3 task took 3.00 seconds, and the fifth trial took 2.14 seconds. In the seeding task, the first trial took 7.50 seconds and the fifth trial took 5.00 seconds. In the watering task, the first trial took 3.75 seconds and the fifth trial took 3.00 seconds. In all three tasks, the time required was shortened with successive trials. In addition, in all trials, the time required to execute the FAB 3 task was the shortest, and the time required to execute the seeding task was the longest. 


\subsection{Comparison of Maximum Oxy-Hb Values among Three Tasks}

In the first and the second trials, the Steel-Dwass test showed no significant differences between the maximum Oxy-Hb values of any two tasks. In the third trials, the $\mathrm{Oxy}-\mathrm{Hb}$ value during the seeding task was significantly greater than during the FAB 3 task $(t=2.351, p=0.049)$, and the Oxy-Hb value during the watering task was significantly greater than during the FAB 3 task $(t=2.578, p=$ 0.027 ) at $\mathrm{CH} 7$ (the right medial FP). In the fourth trials, the Oxy-Hb values during the watering task were significantly greater than the FAB 3 task $(t=$ 2.660, $p=0.021)$ at $\mathrm{CH} 7$ and $\mathrm{CH} 10$ (the left medial FP) $(t=2.392, p=0.044)$. In the fifth trials, the Oxy-Hb value during the seeding task was significantly greater than during the FAB 3 task $(t=2.784, p=0.015)$ and the value during the watering task was significantly greater than during the FAB 3 task $(t=2.578, p=$ $0.027)$ at $\mathrm{CH} 7$; the $\mathrm{Oxy}-\mathrm{Hb}$ value during the seeding task was significantly greater than during the FAB 3 task $(t=2.371, p=0.047)$ and the value during the watering task was greater than during the FAB 3 task at the marginally significant level $(t=2.310, p=0.055)$ at $\mathrm{CH} 10$ (Figure 4). The above results are summarized in Table 3. No significant difference in the maximum Oxy-Hb value was observed between the seeding and watering tasks in the medial and lateral FP through all trials; some channels in the medial FP showed that the maximum Oxy-Hb values of the seeding task and/or the watering task were greater than that of the FAB 3 task after the third trial.

Table 3. Comparison of the maximum Oxy-Hb values among three tasks for subsequent trials. At the channels with ${ }^{\star}$, significant differences in Oxy-Hb values were observed between two tasks to be compared $(p<0.05)$.

\begin{tabular}{|c|c|c|c|c|c|c|c|c|c|}
\hline & \multirow{3}{*}{ Trial } & \multicolumn{4}{|c|}{ Right hemisphere } & \multicolumn{4}{|c|}{ Left hemisphere } \\
\hline & & \multicolumn{3}{|c|}{ lateral } & \multirow{2}{*}{$\begin{array}{c}\text { medial } \\
\mathrm{CH} 7\end{array}$} & \multirow{2}{*}{$\begin{array}{l}\text { medial } \\
\mathrm{CH} 10\end{array}$} & \multicolumn{3}{|c|}{ lateral } \\
\hline & & $\mathrm{CH} 4$ & $\mathrm{CH} 5$ & $\mathrm{CH} 6$ & & & $\mathrm{CH} 11$ & $\mathrm{CH} 12$ & $\mathrm{CH} 13$ \\
\hline \multirow{5}{*}{$\begin{array}{c}\text { Seeding } \\
\text { vs } \\
\text { FAB } 3\end{array}$} & 2 nd & & & & & & & & \\
\hline & $3 \mathrm{rd}$ & & & & * & & & & \\
\hline & 4th & & & & & & & & \\
\hline & 5 th & & & & * & * & & & \\
\hline & 2nd & & & & & & & & \\
\hline \multirow{4}{*}{$\begin{array}{c}\text { Watering } \\
\text { vs } \\
\text { FAB } 3\end{array}$} & $3 \mathrm{rd}$ & & & & * & & & & \\
\hline & 4 th & & & & * & * & & & \\
\hline & 5 th & & & & * & $\begin{array}{l}\text { marginally } \\
\text { significant }\end{array}$ & & & \\
\hline & 2nd & & & & & & & & \\
\hline \multirow{3}{*}{$\begin{array}{c}\text { Seeding } \\
\text { vs } \\
\text { Watering }\end{array}$} & $3 \mathrm{rd}$ & & & & & & & & \\
\hline & 4th & & & & & & & & \\
\hline & 5 th & & & & & & & & \\
\hline
\end{tabular}




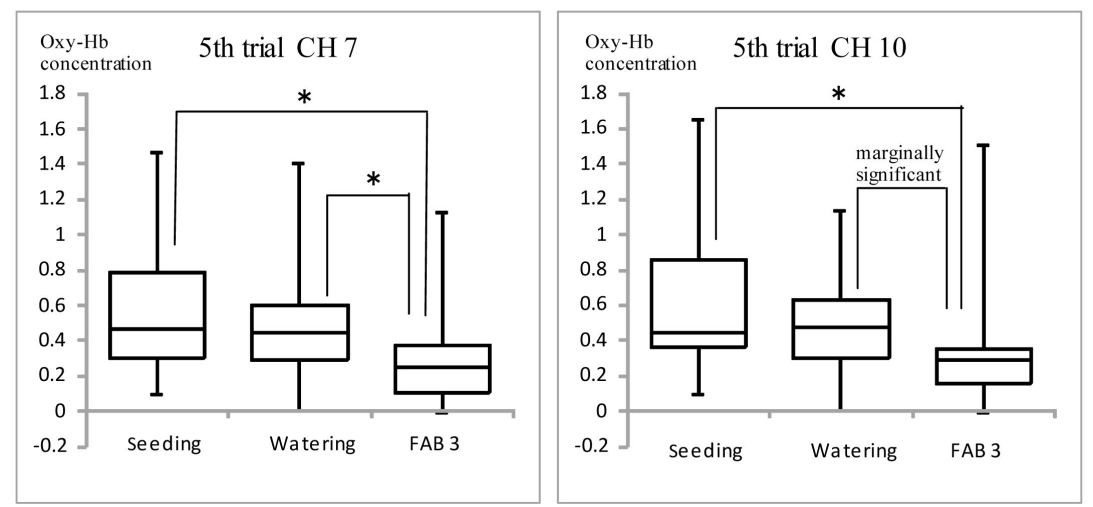

Figure 4. Comparison of maximum Oxy-Hb values in the medial $\mathrm{FP}(\mathrm{CH} 7$ and $\mathrm{CH} 10)$ during the fifth trial. Statistical between-group differences were evaluated by the Steel-Dwass test for nonparametric multiple comparison. Numbers on the longitudinal axis represent $\mathrm{Oxy}-\mathrm{Hb}$ concentration in $\mathrm{mmol} \cdot \mathrm{mm} / 0.1 \mathrm{~s}:{ }^{*} p<0.05$.

\subsection{Comparison of Maximum 0xy-Hb Values among Five Trials within Each Task}

According to the results of the Shirley-Williams test at the significance level of $5.0 \%$ conducted to evaluate maximum $\mathrm{Oxy}-\mathrm{Hb}$ values among five trials, the seeding task did not show significant differences among five trials at any channels which we focused on (CHs 4, 5, 6, 7 in the right hemisphere and CHs 10, 11, 12,13 in the left hemisphere). That is, activation in the medial and lateral FP was sustained in the seeding task, in spite of the increased number of trials.

The watering task did not show significant differences in Oxy-Hb values among five trials at $\mathrm{CH} 7$ (the right medial FP) and $\mathrm{CH} 10$ (the left medial FP), but showed significant decrease at $\mathrm{CH} 5$ (the right lateral FP) and $\mathrm{CH} 12$ (the left lateral FP). At $\mathrm{CH} 5$, the value in the first trial was significantly greater than the values in the second trial $(t=1.959$, critical value $=1.645)$, the third trial $(t=$ 1.883 , critical value $=1.716)$, the fourth trial $(t=1.876$, critical value $=1.739)$ and the fifth trial $(t=1.857$, critical value $=1.750)$. At $\mathrm{CH} 12$, the value in the first trial was significantly greater than the values in the third trial $(t=2.138$, critical value $=1.716)$, the fourth trial $(t=1.803$, critical value $=1.739)$ and the fifth trial $(t=1.835$, critical value $=1.75)$.

The FAB 3 did not show significant differences in Oxy-Hb values among five trials at $\mathrm{CH} 7$ (the right medial FP) and $\mathrm{CH} 10$ (the left medial FP), but showed a significant decrease at $\mathrm{CH} 5$ (the right lateral FP) and $\mathrm{CH} 11$ (the left lateral FP). The value in the first trial was significantly greater than the values in the fourth trial $(t=2.544$, critical value $=1.739)$ and the fifth trial $(t=1.969$, critical value $=$ $1.750)$ at $\mathrm{CH} 5$, and the value in the first trial was significantly greater than the values in the fourth trial $(t=2.342$, critical value $=1.739)$ and the fifth trial $(t=$ 1.826 , critical value $=1.750)$ at $\mathrm{CH} 11$.

The above results are summarized in Table 4 and Figure 5. No significant decrease in the Oxy-Hb values in the three tasks was observed in the medial FP, while some channels in the lateral FP showed significant decrease in the watering task and FAB 3 with the increased number of trials. 

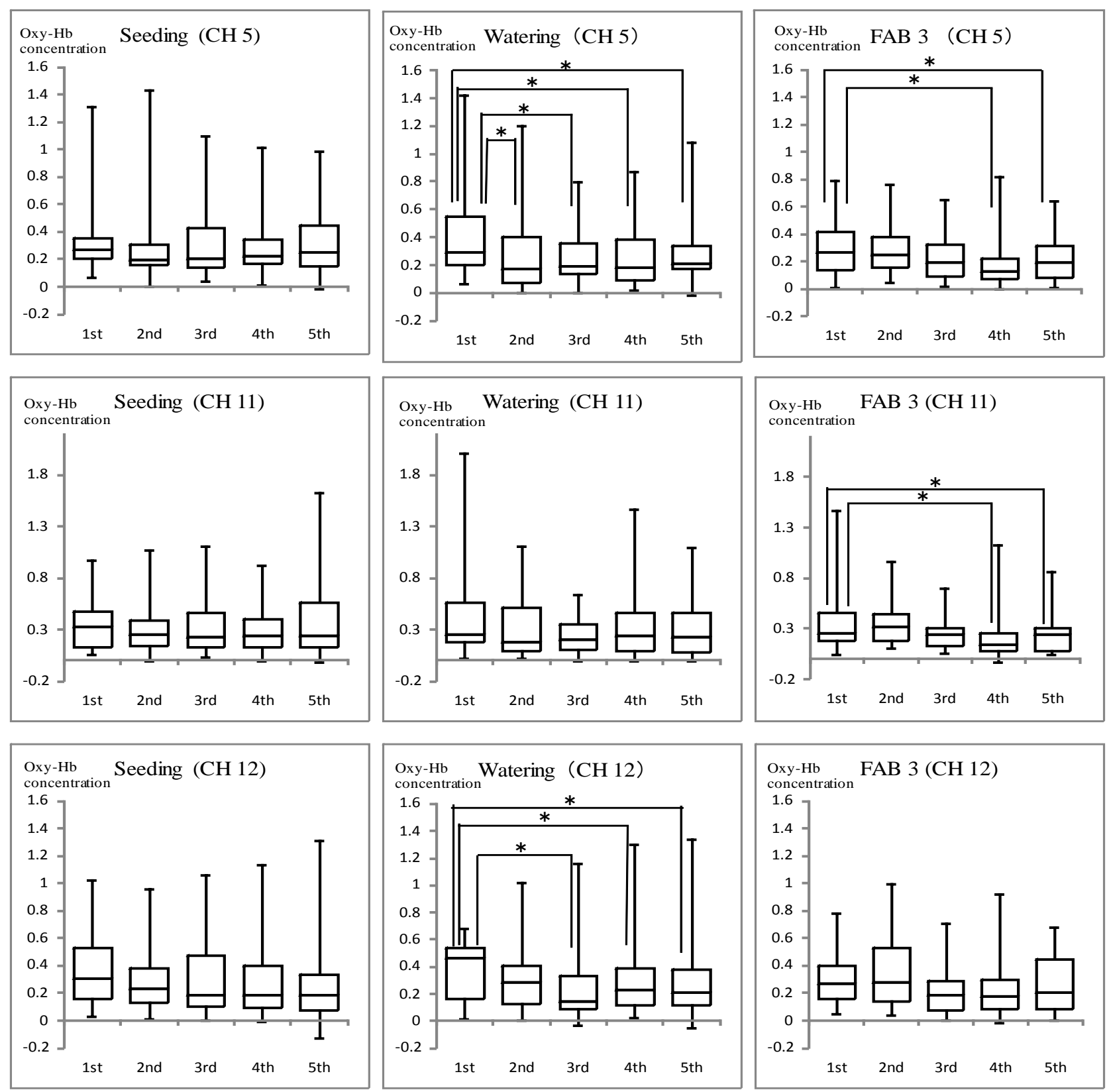

Figure 5. Comparison of maximum Oxy-Hb values at the channels that showed significant changes with the increased number of trials. Statistical differences were evaluated by the Shirley-Williams test for nonparametric multiple comparison. Numbers on the longitudinal axis represent $\mathrm{Oxy}-\mathrm{Hb}$ concentration in $\mathrm{mmol} \cdot \mathrm{mm} / 0.1 \mathrm{~s}:{ }^{*} p<0.05$.

\section{Discussion}

\subsection{Direction of Participants' Eyes during Task Execution}

All the participants looked at their hands and/or the task materials while performing the gardening tasks, but did not typically do so while performing the FAB 3 task. Because participants had practiced each task following the oral explanation and demonstration by the examiner, they had already memorized each procedure prior to the start of the first trial. Nonetheless, to perform the two gardening tasks successfully, the participants needed both the somatosensory 
Table 4. Comparison of the maximum Oxy-Hb values in the first trial vs. trials after the second through fifth trials within each task. At the channels with ${ }^{\star}$, the t-value surpassed the critical value, which indicated significant decrease in Oxy-Hb values compared with those in the first trial.

\begin{tabular}{|c|c|c|c|c|c|c|c|c|c|}
\hline \multirow{3}{*}{ Tasks } & \multirow{3}{*}{ Trial } & \multicolumn{4}{|c|}{ Right hemisphere } & \multicolumn{4}{|c|}{ Left hemisphere } \\
\hline & & \multicolumn{3}{|c|}{ lateral } & \multirow{2}{*}{$\begin{array}{c}\text { medial } \\
\mathrm{CH} 7\end{array}$} & \multirow{2}{*}{$\begin{array}{l}\text { medial } \\
\mathrm{CH} 10\end{array}$} & \multicolumn{3}{|c|}{ lateral } \\
\hline & & $\mathrm{CH} 4$ & CH 5 & $\mathrm{CH} 6$ & & & CH 11 & $\mathrm{CH} 12$ & $\mathrm{CH} 13$ \\
\hline \multirow[t]{4}{*}{ Seeding } & 2nd & & & & & & & & \\
\hline & $3 \mathrm{rd}$ & & & & & & & & \\
\hline & 4 th & & & & & & & & \\
\hline & 5 th & & & & & & & & \\
\hline \multirow[t]{4}{*}{ Watering } & 2nd & & * & & & & & & \\
\hline & $3 \mathrm{rd}$ & & * & & & & & * & \\
\hline & 4th & & * & & & & & * & \\
\hline & 5 th & & * & & & & & * & \\
\hline \multirow[t]{4}{*}{ FAB 3} & 2nd & & & & & & & & \\
\hline & $3 \mathrm{rd}$ & & & & & & & & \\
\hline & 4th & & * & & & & * & & \\
\hline & 5 th & & * & & & & * & & \\
\hline
\end{tabular}

information related to hand motions and the visual information on cells, the soil, seeds, and water. In contrast, visual information was not necessarily required to perform the FAB 3 task, after participants had learned the order of the hand motions (fist-edge-palm). This suggests that one possible difference between the gardening tasks and the FAB 3 task might be the presence or absence of the need for eye-hand coordination.

\subsection{Task Performance and Max Oxy-Hb Values}

In all three tasks, the number of repetitions of the task within 15 seconds significantly increased after the third trial $(p<0.05)$, which indicates that the required time to complete a single series of the process decreased, compared with the first trial. It is speculated that participants were groping for smooth and successful movements to accomplish the task during the first and the second trials; however, they had mastered the procedure well enough to perform the task smoothly during the third trial, and the performance was improved and maintained.

Although a decrease in maximum Oxy-Hb values was observed at some channels in the lateral FP with the increased number of trials in the FAB 3 and the watering tasks, the level of activation surpassed the baseline at every channel during all trials in all three tasks (Figure 4). The fact that a certain degree of FP activation was induced at all channels throughout all five trials indicates that 
some ongoing FP activities were needed, even after participants had learned the rules of the three motor programming tasks well enough, and their task performance had improved. Let us discuss the reasons by looking separately at the medial FP and the lateral FP.

\subsection{FP Activation by Regions}

Before discussing FP activation by regions, it is necessary to clarify the meanings of "attention" and "thought" in these tasks. In order to perform the three tasks, we deemed that the following series of brain activities is repeated: SO attention (attending to somatosensory and/or visual information), SO thought (thought for appropriate task execution based on the external information), SI attention (attending to internal thought for the subsequent procedure), and SI thought (thought for recalling and judging the next action).

\subsubsection{Activation in the Medial FP}

In all tasks, no significant changes in activation were observed at $\mathrm{CH} 7$ (the right medial FP) or $\mathrm{CH} 10$ (the left medial FP), in spite of the increased number of trials. As common features of the three tasks that induced continuous activation in the medial FP, we assume that the participants needed to recall the subsequent action, checking the ongoing motion and/or the task situation as they performed the task at their own pace. In order to check the present situation during task performance, sensory information from the sensory system is necessary. Burgess et al. described a function of medial BA10 [Brodmann area 10] as "biasing attention toward current sensory input" [19]. SO cognitive process (SO attention and SO thought) necessary to perform the tasks is assumed to be one of the causes of the medial FP activation continuously repeated to the fifth trial. Of course, SI cognitive process (SI attention and SI thought) is also deemed to be related together, as Gilbert et al. noted that rostromedial PFC activity is associated with performing tasks that involve both SO and SI thought, compared with tasks involving SI thought alone [25].

In addition, Shimoda et al. found greater activation in the FP corresponding to the medial FP during task performance at the participant's preferred slow pace (PSP), compared with task performance at externally triggered metronome slow pace (MSP). They mentioned that during the PSP task, the subjects were making a move while receiving feedback from their own input systems such as visual processing and motor effector systems; they posited that the FPPFC [the frontopolar cortex, BA 10] is specifically involved in evaluating self-generated responses or plans for action [26].

The results of comparison of maximum Oxy-Hb values across the three tasks at the fifth trial showed significantly greater activation during the seeding task than during the FAB 3 task at $\mathrm{CH} 7$ and $\mathrm{CH} 10(p<0.05)$, and significantly greater activation during the watering task than during the FAB 3 task at $\mathrm{CH} 7$ $(p<0.05)$, which was marginally significant at $\mathrm{CH} 10(p=0.055)$. The three tasks have common characteristics, in that they require SI and SO attention to 
accomplish the tasks, and that the participants can execute the tasks at their own pace. Let us now attempt to discuss what caused the differences in activation between the gardening tasks and the FAB 3 task, from two points of view: 1) the number of sensory information sources, and 2) the number of objects to which the participants had to pay SO attention. The first factor is that both somatosensory information and visual information were necessary in executing the seeding task and the watering task. In contrast, as $79.2 \%$ of the participants performed the task without looking at their hands, visual information was not necessarily needed to perform the FAB 3 task, which could be carried out with somatosensory information only.

The second factor is the difference in the number of objects to which the participants had to pay SO attention. Every time SO attention takes place, SO thoughts arise. In the FAB 3 task, participants pay their $\mathrm{SO}$ attention to three hand shapes (fist, edge, and palm). Meanwhile, to sow a single seed in a cell, the participants pay their SO attention to nine objects in order (i.e. a cell tray with 25 cells connected in a matrix state, a cell to be seeded, soil in the cell, a hole in the center of the cell, a saucer of seeds, seeds, a hole to put a seed in, the seed put into the hole, the soil to cover and be pressed over the seed). In watering, the participants pay their SO attention to six objects (i.e. a plastic bottle, a cell tray with 25 cells connected in a matrix state, a cell to be watered, the soil in the cell, running water out of the plastic bottle, and the level of water in the cell). It is speculated that the greater medial FP activation in the gardening tasks might have been due to the larger number of sensory information sources to which participants had to pay attention, and the larger number of SO attention and thoughts, compared with the FAB 3 task.

\subsubsection{Activation in the Lateral FP}

In comparing maximum Oxy-Hb values across the three tasks, no significant differences were observed in spite of the increased number of trials, for all three tasks. On the other hand, the watering task and the FAB 3 task showed a significant decrease in a portion of the lateral FP, with an increased number of trials. This indicates that a significant difference between the seeding task and the watering task, or between the seeding task and the FAB 3 task in the lateral FP may be shown if the number of trials is increased.

It was reported by Gillbert et al. that the right lateral FP shows transient activity by switching between two phases (SO and SI thought), regardless of the direction of the switch. They also explained that "external" phases still required some degree of SI thought to perform the tasks [25]. In contrast to the tasks in their study, our research tasks were not designed to repeat the external phase and the internal phase alternately, but were executed based on the external stimuli (i.e. external somatosensory and/or visual information). In our study, it is speculated that SI thought was needed to recall and/or make decisions about what action needed to be taken, each time somatosensory and/or visual stimulus changed with progression of the process. Iteration of SO cognitive process and 
SI cognitive process in the three tasks in our study has the possibility to have led to the right lateral FP activation. Furthermore, Pollmann reported selectively increased dimension change-related activation in the left frontopolar cortex for stimulus-driven visual dimension weighting [27]. The above-mentioned two factors-switching between SI attention and SO attention, and change of visual dimension weighting-were also necessary to execute three tasks in our study, which could induce bilateral activation in the lateral FP.

In the seeding task, no significant decrease in activation in the lateral FP was observed with an increase in the number of trials. However, the watering task showed a significant decrease at $\mathrm{CH} 5$ (the right hemisphere) after the second trial and at $\mathrm{CH} 12$ (the left hemisphere) after the third trial. The FAB 3 task showed a significant decrease at $\mathrm{CH} 5$ (the right hemisphere) and at $\mathrm{CH} 11$ (the left hemisphere) after the fourth trial $(p<0.05)$. Three reasons are speculated for the transient activation continuously repeated to the last trial in the seeding task. First, the number of objects to which the participants shifted their attention was the largest in the seeding task, in which switching between SI attention and SO attention was conducted most frequently. The participants made each move by recalling the subsequent motion with sensory feedback from visual and/or somatosensory input related to the objects. Every time the participants went forward and shifted attention from one object to another, switching between two kinds of attention was necessary. Second, changes in visual dimension weighting were most frequent during the seeding task, starting from: 1) location (one cell among twenty-five connected cells), shifting to 2) location (the center of the soil in the work cell), 3) space (depth of a hole in the soil), 4) location (a saucer), 5) shape (seeds), 6) number (one seed among seeds on the saucer), 7) space (the hole in the work cell), 8) volume (the soil to cover the seed with), to 9) space (the hole filled and pressed down with soil). Considering that it took approximately five seconds for the participants to sow a bean seed in the fifth trial, nine changes in visual dimension weighting were necessary within a mere five seconds during the seeding task. In contrast, changes in visual dimension weighting during the watering task arose between location (one cell among twenty-five connected cells) and volume (rise and fall of the water level in the cell), while the visual dimension involved in the FAB 3 task was only shape (three kinds of hand shapes). That is, decreased activation in the right lateral FP in the watering and FAB 3 tasks appears to be related to the smaller number of times switching between SI attention and SO attention, compared with the seeding task. Decreased activation in the left lateral FP in the watering task and FAB 3 appears to be caused by the smaller number of changes of visual dimension weighting and reallocation of attentional resources. Finally, each trial of the seeding task and the watering task included inevitable fluctuating factors compared with the FAB 3 task. The fluctuating factors in the seeding task were: 1) shifting location of a work cell with progression of work, 2) depth of the seeding hole dependent on strength of a finger, and 3) easiness of picking up a seed, dependent on the number and location of seeds remaining on the saucer. In the case of the watering task, the fac- 
tors were: 1) shifting location of the work cell, and 2) the remaining amount of water in the plastic bottle with the progression of work. The decreasing amount of water affects water pressure, and the participants needed to adjust the tilt of the plastic bottle. The FAB 3 task has no such fluctuating factors. In the Introduction section, we mentioned the findings by Strange, et al. [11] that after the FPPC has learned a rule, the more posterior areas mediate rule application. Although according to their findings, repetition of the same tasks might not lead to continuing activation in the same brain area, in our study the gardening tasks were found to induce continual FP activation. It is possible that the fluctuating factors characteristically encountered in ordinary gardening tasks may play some additional role in such continual FP activation, even after the areas of activation have shifted from the FP (for learning task rules) to more posterior areas (for rule application).

\section{Conclusions}

The results of the present study lead to the following characteristics of gardening activities that appear to be closely related to FP activation:

1) Two types of sensory information are involved-visual information and somatosensory information.

2) Input of a diversified range of SO stimulation is necessary to complete the work.

3) Switching between $\mathrm{SO}$ and SI attention is frequent.

4) Visual dimension weighting changes occur frequently during the process (i.e. the dimension of the objects to which the participant must pay attention changes at each step in the process.)

5) External environmental changes arise inevitably as a series of gardening processes are repeated.

It is presumed that the first and second gardening characteristics listed above may be conducive to the medial FP activation. The third and fourth characteristics may be conducive to activation of the right lateral FP and to activation of the left lateral FP, respectively. In addition, it is possible that the fifth characteristic may contribute to continual FP activation both in the medial and lateral FP.

In the present study, participants needed to respond to changes in the external work environment in order to execute the gardening tasks successfully. We assume that this necessity for collation of external information and correspondence to changes in the external environment required function of the medial and lateral FP and induced repeated and continuous FP activation in the gardening tasks. The impact of this feature of gardening on FP activation appeared prominently in the seeding task.

Findings from previous epidemiological studies suggest that participation in gardening activities could be an effective strategy for dementia prevention; this study helps confirm previous findings. Implementation of many gardening activities in addition to seeding and watering (e.g. thinning, setting out seedlings, harvesting, deadheading, weeding, plowing with a spade, etc.) requires "eye-hand 
coordination" and/or "dexterity of fingers, hands, and/or body". Moreover, the frequent switching between SO attention and SI attention necessary for such gardening work would activate the medial FP and the lateral FP. Because gardening activities include daily and routine care of plants, which exhibit individual differences and growth patterns, gardening activities inherently require changes in behavior corresponding to changes in the plants. This need for taking a flexible approach according to changes of the external circumstances caused by the plants can play an important role in FP activation.

Gardening can easily provide continual opportunities to use physical functions, motivated by the growth of plants. Moreover, many gardening activities are easy to do, even for older adults with declined cognitive and/or physical function, for the following reasons: 1) The process of many gardening activities is easy to understand by watching the procedure without detailed explanation; 2) Many gardening activities use procedural memory that people have already learned in everyday life experience; 3 ) The degree of difficulty of the work is easily adjustable by using plant material at different growing stages (seeds, bulbs, or seedlings) and/or of different sizes; and 4) A person's working position can be adjusted by using containers and raised beds to accommodate their level of physical function. These characteristics of gardening activities have the possibility to induce FP activation on a daily basis, and contribute to the suppression of cognitive decline. The findings of this study suggest that certain gardening tasks are especially effective in activating specific parts of the brain and inducing sustained activation, thus providing a clue to how daily gardening activities could become a useful tool in the prevention of dementia.

\section{Acknowledgements}

This study was supported by Grant-in-Aid for Scientific Research C 15K07298 to Masahiro Toyoda by Japan Society for the Promotion of Science.

\section{References}

[1] Cherry, K.E., Park, D.C., Frieske, D.A. and Smith, A.D. (1996) Verbal and Picotorial Elaborations Enhance Memory in Young and Older Adults. Aging, Neuropsychology, and Cognition, 3, 15-29. https://doi.org/10.1080/13825589608256609

[2] Kwee, I.L. and Nakada, T. (2003) Dorsolateral Prefrontal Lobe Activation Declines Significantly with Age-Functional NIRS Study. Journal of Neurology, 250, 525-529. https://doi.org/10.1007/s00415-003-1028-x

[3] Zinke, K., Zeintl, M., Rose, N.S., Putzmann, J., Pydde, A. and Kliegel, M. (2014) Working Memory Training and Transfer in Older Adults: Effects of Age, Baseline Performance, and Training Gains. Developmental Psychology, 50, 304-315. https://doi.org/10.1037/a0032982

[4] Belleville, S., Clément, F., Mellah, S., Gilbert, B., Fontaine, F. and Gauthier, S. (2011) Training-Related Brain Plasticity in Participants at Risk of Developing Alzheimer's Disease. Brain, 134, 1623-1634. https://doi.org/10.1093/brain/awr037

[5] Fabrigoule, C., Letenneur, L., Dartigues, J.F., Zarrouk, M., Commenges, D. and Barberger-Gateau, P. (1995) Social and Leisure Activities and Risk of Dementia: A 
Prospective Longitudinal Study. Journal of the American Geriatrics Society, 43, 485-490. https://doi.org/10.1111/j.1532-5415.1995.tb06093.x

[6] Simons, L.A., Simons, J., McCallum, J. and Friedlander, Y. (2006) Lifestyle Factors and Risk of Dementia: Dubbo Study of the Elderly. Medical Journal of Australia, 184, 68-70.

[7] Jedrziewski, M.K., Ewbank, D.C., Wang, H. and Trojanowski, J.Q. (2010) Exercise and Cognition: Results from the National Long Term Care Survey. Alzheimers Dementia, 6, 448-455.

[8] Toyoda, M., Yokota, Y. and Rodiek, S. (2016) A Motor Programming Task Activates the Prefrontal Cortex More than a Sensitivity-to-Interference Task or an Inhibitory Control Task in Older Adults. Journal of Behavioral and Brain Science, 6, 433-447. https://doi.org/10.4236/jbbs.2016.611040

[9] Dubois, B., Slachevsky, A., Litvan, I. and Pillon, B. (2000) The FAB: A Frontal Assessment Battery at Bedside. Neurology, 55, 1621-1626. https://doi.org/10.1212/WNL.55.11.1621

[10] Toyoda, M. and Yokota, Y. (2016) The Effects of Gardening Activities on Prefrontal Area Measured with Near-Infrared Spectroscopy (NIRS). Acta Horticulturae, 1121, 33-38. https://doi.org/10.17660/ActaHortic.2016.1121.6

[11] Strange, B.A., Henson, R.N.A., Friston, K.J. and Dolan, R.J. (2001) Anterior Prefrontal Cortex Mediates Rule Learning in Humans. Cerebral Cortex, 11, 1040-1046. https://doi.org/10.1093/cercor/11.11.1040

[12] Turner, M.S., Simons, J.S., Gilbert, S.J., Frith, C.D. and Burgess, P.W. (2008) Distinct Roles for Lateral and Medial Rostral Prefrontal Cortex in Source Monitoring of Perceived and Imagined Events. Neuropsychologia, 46, 1442-1453.

[13] Buckner, R.L. (1996) Beyond HERA: Contributions of Specific Prefrontal Brain Areas to Long-Term Memory Retrieval. Psychonomic Bulletin and Review, 3, 149-158. https://doi.org/10.3758/BF03212413

[14] Burgess, P.W., Quayle, A. and Frith, C.D. (2001) Brain Regions Involved in Prospective Memory as Determined by Positron Emission Tomography. Neuropsychologia, 39, 545-555.

[15] Christoff, K. and Gabrieli, J.D.E. (2000) The Frontopolar Cortex and Human Cognition: Evidence for a Rostrocaudal Hierarchical Organization within the Human Prefrontal Cortex. Psychobiology, 28, 168-186. https://link.springer.com/article/10.3758/BF03331976

[16] Koechlin, E., Basso, G., Pletrini, P., Panzer, S. and Grafman, J. (1999) The Role of the Anterior Prefrontal Cortex in Human Cognition, Nature, 399, 148-151. https://search.proquest.com/openview/31e428d7c7dcd64217b00d5beed6b55d/1.pdf ?pq-origsite $=$ gscholar $\& \mathrm{cbl}=40569$ https://doi.org/10.1038/20178

[17] Raiche, M.E., MacLeod, A.M., Snyder, A.Z., Powers, W.J., Gusnard, D.A. and Shulman, G.L. (2001) A Default Mode of Brain Function. Proceedings of the National Academy of Sciences, 98, 676-682. https://doi.org/10.1073/pnas.98.2.676

[18] Duncan, J., Phillips, L. and McLeod, P. (2005) Measuring the Mind: Speed, Control, and Age. Oxford University Press, Oxford, 217-248.

[19] Burgess, P.W., Dumontheil, I. and Gilbert, S.J. (2007) The Gateway Hypothesis of Rostral Prefrontal Cortex (Area 10) Function. Trends in Cognitive Sciences, 11, 290-298.

[20] Fletcher, P.C. and Henson, R.N.A. (2001) Frontal Lobes and Human Memory: In- 
sights from Functional Neuroimaging. Brain, 124, 849-881.

https://doi.org/10.1093/brain/124.5.849

[21] Treitz, F.H., Heyder, K. and Daum, I. (2007) Differential Course of Executive Control Change during Normal Aging. Aging, Neuropsychology, and Cognition, 14, 370-393. https://doi.org/10.1080/13825580600678442

[22] Pivik, R.T., Broughton, R.J., Coppola, R., Davidson, R.J., Fox, N. and Nuwer, M.R. (1993) Guidelines for Recording and Quantitative Analysis of Electroencephalo-Graphic Activity in Research Contexts. Psychophysiology, 30, 547-558.

http://onlinelibrary.wiley.com https://doi.org/10.1111/j.1469-8986.1993.tb02081.x

[23] Hoshi, Y., Kobayashi, N. and Tamura, M. (2001) Interpretation of Near-Infrared Spectroscopy Signals: A Study with a Newly Developed Perfused Rat Brain Model. Journal of Applied Physiology, 90, 1657-1662.

http://jap.physiology.org/content/90/5/1657.short

[24] Sakatani, K., Xie, Y., Lichty, W., Li, S. and Zuo, H. (1998) Language-Activated Cerebral Blood Oxygenation and Hemodynamic Changes of the Left Prefrontal Cortex in Poststroke Aphasic Patients a Near-Infrared Spectroscopy Study. Stroke, 29, 1299-1304. http://stroke.ahajournals.org/content/29/7/1299.short https://doi.org/10.1161/01.STR.29.7.1299

[25] Gilbert, S.J., Frith, C.D. and Burgess, P.W. (2005) Involvement of Rostral Prefrontal Cortex in Selection between Stimulus-Oriented and Stimulus-Independent Thought. European Journal of Neuroscience, 21, 1423-1431. https://doi.org/10.1111/j.1460-9568.2005.03981.x

[26] Shimoda, K., Moriguchi, Y., Tsuchiya, K., Katsuyama, S. and Tozato, F. (2014) Activation of the Prefrontal Cortex while Performing a Task at Preferred Slow Pace and Metronome Slow Pace: A Functional Near-Infrared Spectroscopy Study. Neural Plasticity, 2014, Article ID: 269120. https://doi.org/10.1155/2014/269120

[27] Pollmann, S. (2004) Anterior Prefrontal Cortex Contributions to Attention Control. Experimental Psychology, 51, 270-278. https://doi.org/10.1027/1618-3169.51.4.270 